\title{
Stub-Loaded Resonator-Fed Filtering Patch Antenna with Improved Bandwidth
}

\author{
Chunxu Mao, Steven Gao \\ School of Engineering and Digital Arts \\ University of Kent \\ Canterbury, United Kingdom
}

\author{
Yi Wang \\ Department of Engineering Science \\ University of Greenwich \\ Chatham, United Kingdom
}

\begin{abstract}
A method of designing a patch antenna with wideband and filtering characteristics is proposed in this paper. Different from traditional aperture-coupled patch antenna, a stub loaded resonator (SLR) is employed as the feed. This coupled SLR-patch produces three reflection zeros (dual-mode from the SLR and one from the patch) and therefore a wider bandwidth. Due to the resonant property of the SLR, the antenna exhibits a filtering performance and good out-of-band rejection. This integration approach eliminates the $50 \mathrm{Ohm}$ interfaces between the traditionally cascaded filter and antenna. This contributes to a more compact and simplified RF frontend. Methods to control the modes of the SLR are investigated. The simulation and measurement results agree well with each other, showing an excellent performance in terms of bandwidth, frequency selectivity and radiation patterns.
\end{abstract}

Keywords-Filter, stub-loaded resonator, patch antenna, bandwidth.

\section{INTRODUCTION}

Modern wireless communications demand the RF frontend to be more compact and multifunctional. Integration of passive microwave components such as antennas, filters and power dividers provides a feasible solution to meet the requirements. Traditionally, the differences in the design theories and approaches lead to the division of "antenna engineering" and "microwave engineering". Although this independent design approach facilitates the modularization of the system and reduce the complexity, it leads to increased volume of RF frontend and deterioration of the frequency response as mismatch between the filters and antennas often occur. Using the integrated filter-antenna design approach, the $50 \mathrm{Ohm}$ interfaces can be eliminated and the frontend size could be reduced significantly. On the other hand, the systematic synthesis methods of filters can be applied to antenna design in order to improve the bandwidth, frequency selectivity and insert loss of the RF frontend. In the past few years, several works related to filter-antenna integration were reported. In [1], a bandpass filter and an antenna were integrated for miniaturisation. The integration of filter and antenna could also increase the bandwidth and frequency selectivity when compared with a cascaded system [2], [3]. In addition, the integrated designs could have the merits of higher-order harmonics suppression [4] and flat gain response [5].

Another issue to be concerned with is the relatively narrow bandwidth of the patch antenna, which is usually enhanced by

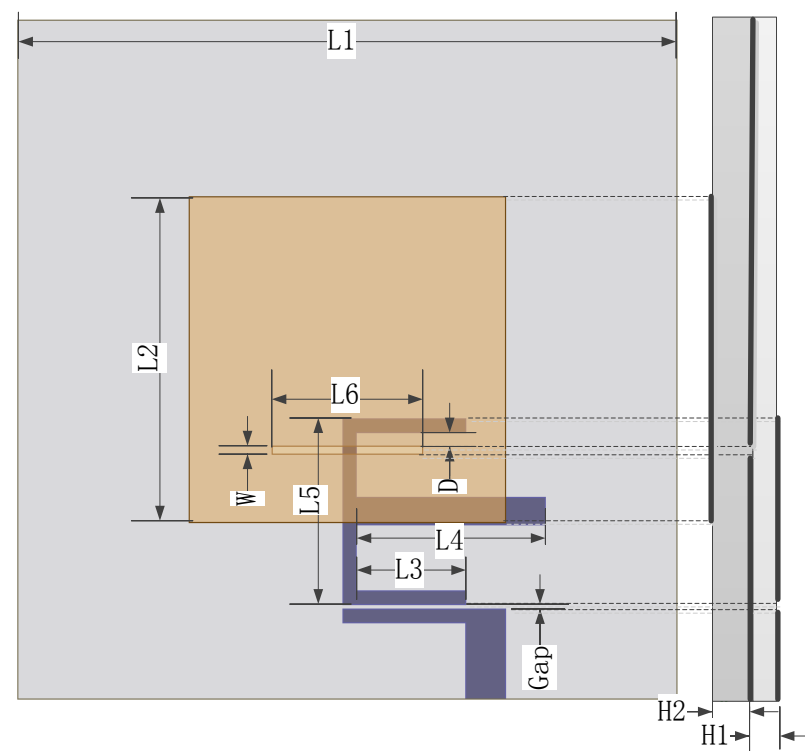

Fig. 1. The configuration of the proposed SLR-fed filtering patch antenna

increasing the thickness of the substrate [6], using stacked configuration [7] or splitting slots in the patch [8]. However, these methods have their limitations. Thick substrates not only increase the risk of unwanted surface waves, but also increase the weight and cost of the component. Stacked configurations require more substrates to implement the parasitic patches, resulting in a higher profile and larger volume. Slots in the patch can greatly increase the bandwidth, but they could also deteriorate the polarization purity. This prohibits the applications when dual polarization or circular polarization are required.

In this paper, a novel integrated filtering antenna is proposed by using a stub-loaded resonator (SLR) to couple to and feed a patch antenna. The modes of the SLR and the patch antenna are investigated and the methods to control the modes are presented. By tuning the lengths of the SLR, the two modes can be located at both sides of the resonant mode of the patch, resulting in an enhanced bandwidth of the patch antenna. Furthermore, the bandwidth can be controlled by adjusting the coupling strength between the SLR and patch. Due to the resonant characteristics of the SLR, the SLR-patch antenna exhibits filtering features with a rapid transition from out-band to in-band. The prototype is fabricated and tested and the measured results agree well with the simulations, showing 


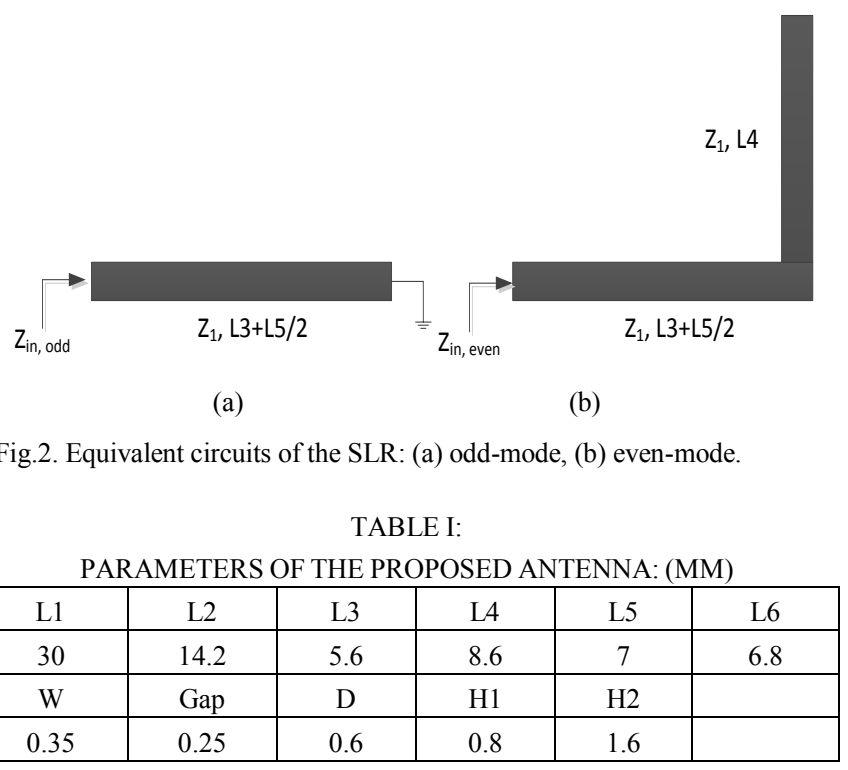

a wideband impedance matching, improved frequency selectivity and radiation patterns.

\section{SLR-FED PATCH ANTENNA}

\section{A. Configuration}

Fig. 1 shows the configuration of the proposed SLR-fed filtering antenna. The antenna is designed to operate at $5 \mathrm{GHz}$. The antenna has a stacked structure. The SLR and the feeding network are printed on the bottom layer of the lower substrate, whereas the square patch is printed on the top layer of the upper substrate. The ground plane is sandwiched between the two substrates, where a slot is cut to enable the coupling between the SLR and the patch. The dimension of the patch is approximately half of a wavelength and the SLR is a dualmode resonator. This stacked structure not only makes the design more compact, but also reduces the adverse interference between the feeding network and the patch antenna. Rogers 4003 substrates with a dielectric constant of 3.55 and a loss tangent of 0.009 are used in the design. The thicknesses of the lower and upper substrates are $0.8 \mathrm{~mm}$ and $1.6 \mathrm{~mm}$, respectively. The design and optimization were performed using High Frequency Simulation Software (HFSS 15) and the optimized parameters are shown in Table I.

\section{B. Design methodology}

The design method for resonator-coupled filters is adopted in this filter-antenna integration scheme. Fig. 2 shows the equivalent circuits of the SLR, where L3, L4 and L5 are defined in Fig. 1. Due to its symmetry, the SLR can be analyzed using odd- and even- mode method [9]. When the odd-mode is excited, the voltage at the middle of the SLR is zero. So the SLR can be treated as being short-circuited at the middle, as shown in Fig. 2(a). When the even-mode is excited, the symmetrical plane can be viewed as a magnetic wall, equivalent to an open end, as depicted in Fig. 2(b). The input impedance for the odd- and even-mode can be derived as

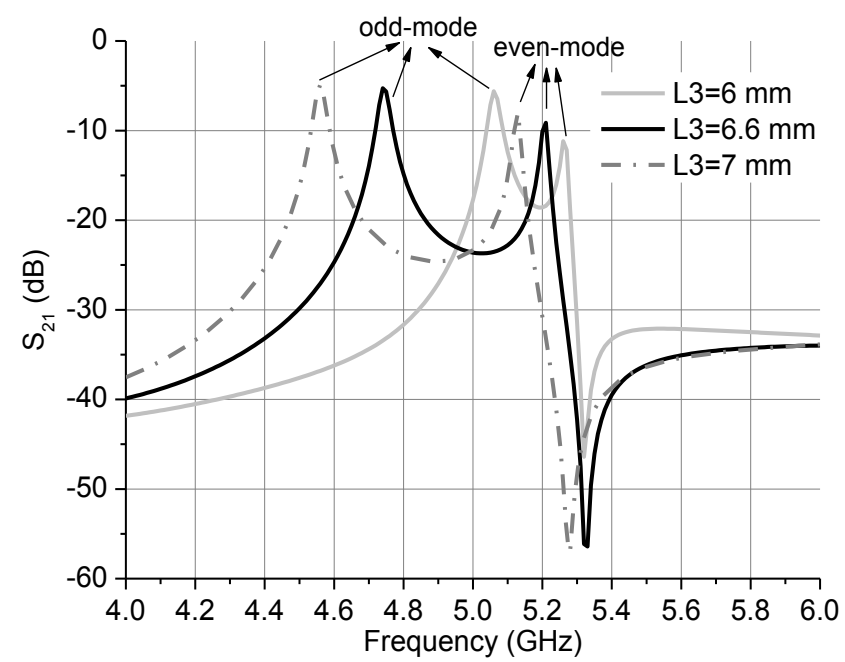

Fig. 3. Variation of the odd- and even-mode of the SLR with $L 3$.

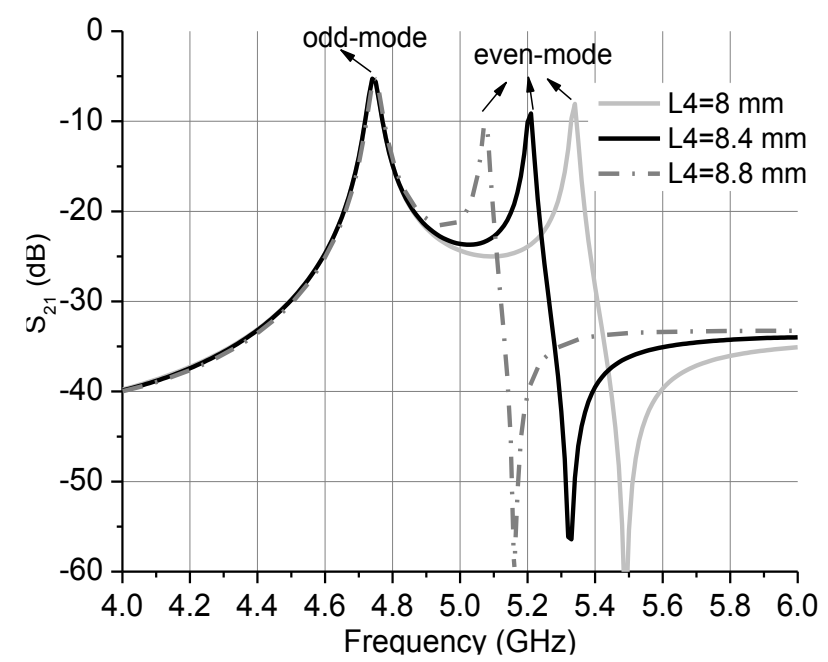

Fig. 4. Variation of the odd- and even-mode of the SLR with L4.

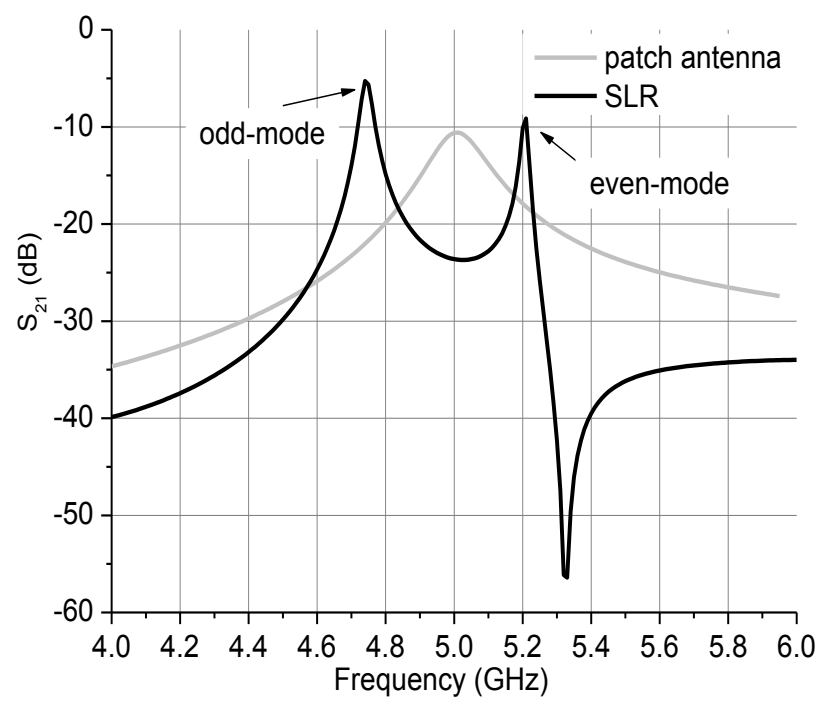

Fig. 5. Resonant modes of the SLR and the patch. 


$$
\begin{aligned}
& Z_{\text {in,odd }}=j Z_{1} \tan \beta(L 3+L 5 / 2) \\
& Z_{\text {in, even }}=-\frac{Z_{1}}{j \tan \beta(L 3+L 5 / 2+L 4)}
\end{aligned}
$$

where $Z_{1}$ is the characteristic impedance of the microstrip and $\beta$ is the transmission constant. When $Z_{\text {in }}$ is infinite, the SLR resonates and the odd- and even-mode resonant frequencies can be derived as

$$
\begin{aligned}
& L 3+L 5 / 2=\frac{\lambda}{4}(2 n-1) \\
& L 3+L 4+L 5 / 2=\frac{n \lambda}{2}
\end{aligned}
$$

where $n=1,2,3 \ldots$ In this design, $n=1$ is chosen. According to (3) and (4), the odd-mode and even-mode resonant frequencies can be controlled by tuning $L 3$ and $L 4$. Fig. 3 shows the change of the odd- and even-mode with $L 3$. It is observed that both modes move towards lower frequencies as $L 3$ increases, but the odd-mode is more affected than the evenmode. From (4), it can be seen that the even-mode can be controlled independently, as shown in Fig. 4. Only the evenmode frequency shifts to lower band when $L 4$ increases. The odd-mode remains unaffected.

Here the microstrip patch antenna is also regarded as a resonator with its fundamental mode at the half wavelength frequency. This single mode resonant characteristic limits its operation bandwidth. In this design, a method of enhancing its bandwidth is demonstrated by assembling the resonant frequencies of the SLR and the patch together, producing a wider combined band. To achieve this goal, the resonant modes of the SLR are adjusted to locate at both sides of the mode of the patch, as shown in Fig. 5. The dual-mode SLR and the patch are electromagnetically coupled together through the slot in the ground plane. By optimizing the strength of the coupling (coupling coefficient), a third-order filtering response is achieved as shown in Fig. 6. Compared with a traditional microstrip-fed patch antenna of a bandwidth of $140 \mathrm{MHz}$, the proposed antenna exhibits a much wider bandwidth of 400 MHz with three reflection zeros. The proposed SLR-patch also exhibits a much sharper skirt and a higher out-of-band rejection.

\section{RESULTS AND DISCUSSION}

The proposed SLR-patch filtering antenna is fabricated and the prototype is shown in Fig. 7. The simulated and measured $\mathrm{S}_{11}$ and antenna gain are shown together in Fig. 8. The simulated $S_{11}$ of the traditional patch antenna is also added for comparison. It is observed that the simulated $S_{11}$ of the proposed antenna agrees well with the measurement with three reflection zeros at 4.8,5.0 and $5.2 \mathrm{GHz}$ clearly identifiable. The fractional bandwidth is over $8 \%$, which is significantly improved when compared with the traditional patch antenna. Furthermore, the antenna also exhibits an excellent filtering performance. The antenna has a flat gain of around $6 \mathrm{dBi}$ from 4.8 to $5.2 \mathrm{GHz}$. Out of the band, the gain rapidly decreases to below $-15 \mathrm{dBi}$ below $4.6 \mathrm{GHz}$ and above $5.3 \mathrm{GHz}$,

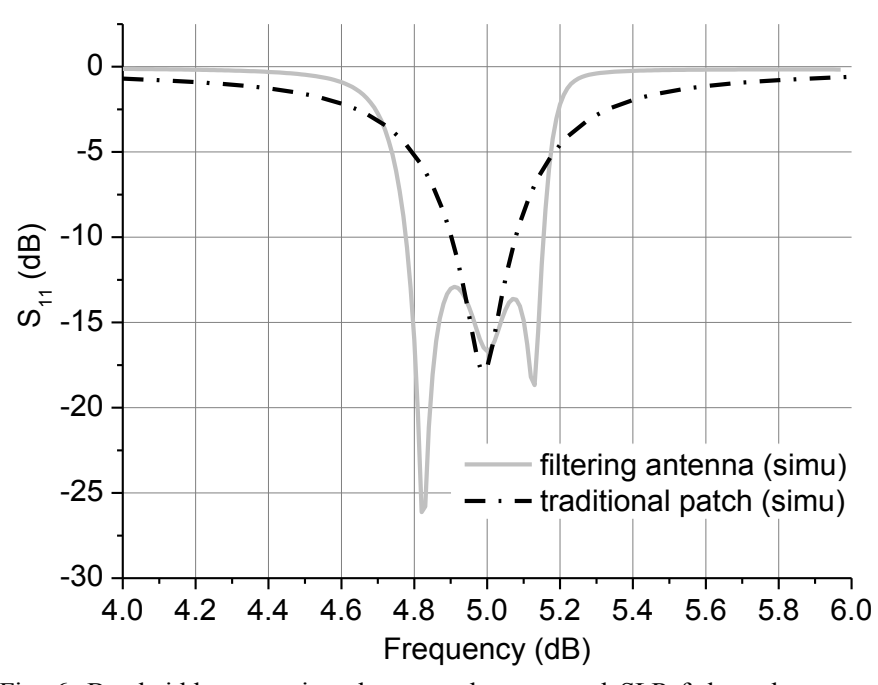

Fig. 6. Bandwidth comparison between the proposed SLR-fed patch antenna and a traditional microstrip-fed patch antenna.

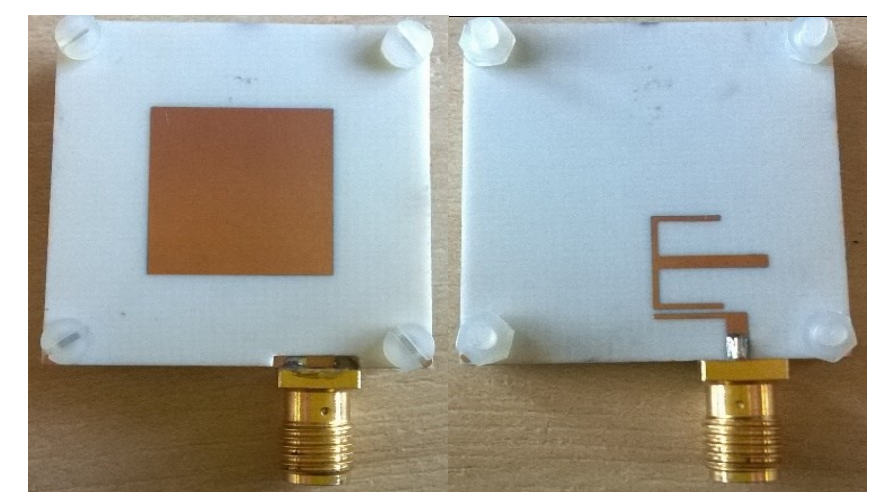

(a)

(b)

Fig. 7. Photograph of the filtering-antenna: (a) the patch, (b) the SLR and the feed line.

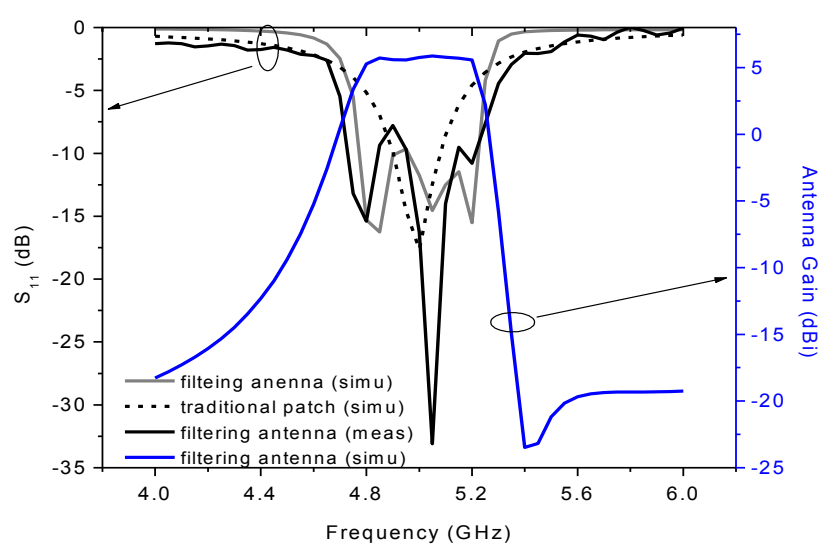

Fig. 8. Simulated and measured $S_{11}$ of the proposed antenna and its simulated gain.

demonstrating that the proposed antenna exhibits an excellent performance in terms of frequency selectivity and out-of-band rejection. 


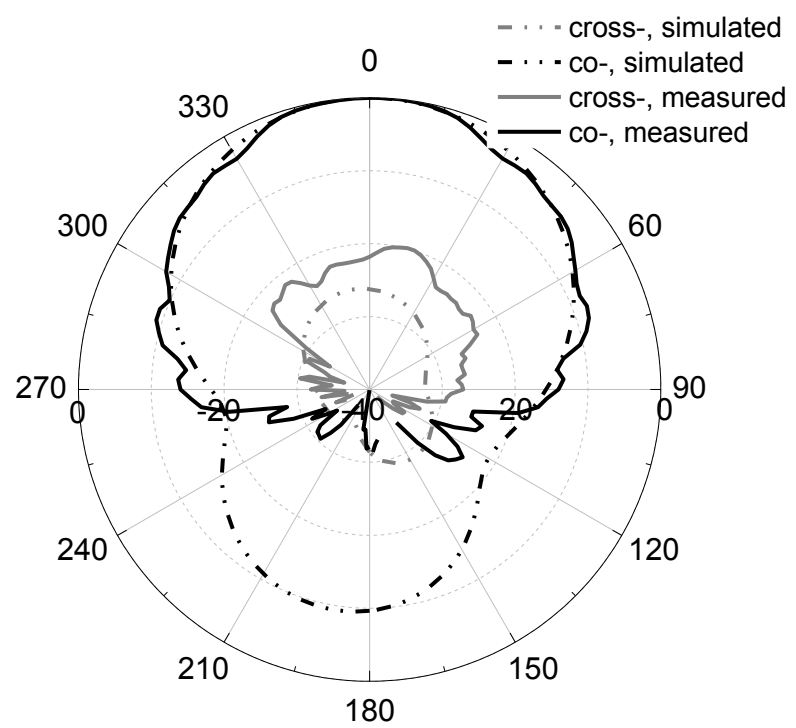

(a)

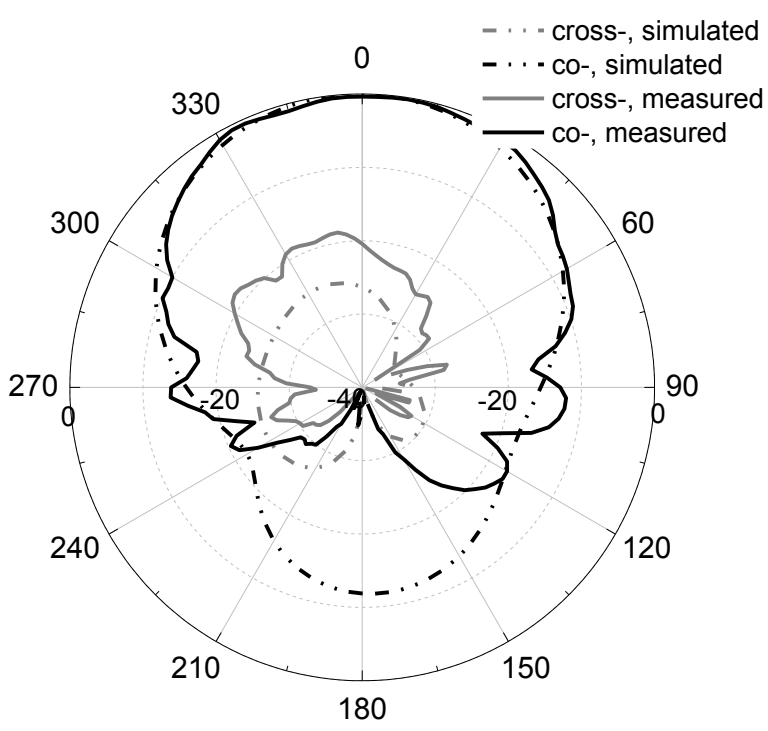

(b)

Fig. 9. Simulated and measured normalized radiation patterns: (a) E-plane, (b) H-plane.

Fig. 9 shows the simulated and measured normalized radiation patterns at $5 \mathrm{GHz}$. It is observed that the antenna shows a directional radiation in the broadside, with a cross polarization better than $20 \mathrm{~dB}$. The measured results agree well with the simulations, except for the backward patterns. This discrepancy is attributed to the scattering from the rotator in the anechoic chamber.

\section{CONCLUSION}

In this paper, a novel SLR-fed patch antenna with improved bandwidth and excellent filtering performance is proposed. The design methodology as well as the modes of the SLR and the patch antenna are investigated. This integrated design improves the bandwidth without increasing the thickness and complexity of the RF frontend. This antenna also shows an improved frequency selectivity without cascading a bandpass filter. Therefore, a multifunctional RF frontend with a compact size can be achieved. Measurement results agree well with the simulated ones, which verifies the design concept.

\section{REFERENCES}

[1] T. L. Nadan, J. P. Coupez, S. Toutain, and C. Person, "Optimization and miniaturization of a filter/antenna multi-function module using a composite ceramic-foam substrate," 1999 IEEE MTT-S Int. Microw. Symp. Dig., pp. 219-222, June 1999.

[2] C. X. Mao, S. Gao, Y. Wang, F. Qin and Q. X. Chu, "Multi-mode resonator-fed dual polarized antenna array with enhanced bandwidth and selectivity," IEEE Trans. Antennas and Propag., vol. 63, no. 12, pp. 5492-5499, Dec. 2015.

[3] C. X. Mao, S. Gao, Z. P. Wang, Y. Wang, F. Qin, B. Sanz and Q. X. $\mathrm{Chu}$, "Integrated Filtering-Antenna with Controllable Frequency Bandwidth," $9^{\text {th }}$ European Conf. on Antenna and Propag., pp. 1-4, 2015.

[4] D. Zayniyev and D. Budimir, "An Integrated Antenna-Filter with Harmonic Rejection," $3^{\text {rd }}$ European Conf. on Antennas and Propag., pp. 393-394, 2009.

[5] C. K. Lin and S. J. Chung, "A Compact Filtering Microstrip Antenna With Quasi-Elliptic Broadside Antenna Gain Response" Antenna Wireless Propag. Lett., vol. 10, pp. 381-384, 2011.

[6] D.Li, P. F. Guo, Q. Dai and Y. Q. Fu, "Broadband Capacitively Coupled Stacked Patch Antenna for GNSS Applications," Antenna Wireless Propag. Lett.,vol. 11, pp. 701-704, 2012.

[7] R. Caso, A. A. Serra, M. R. Pino, P. Nepa and G. Manara, "A Wideband Slot-Coupled Stacked-Patch Array for Wireless Communications," Antenna Wireless Propag. Lett., vol. 9, pp. 986-989, 2010.

[8] W. H. Hsu, K. L. Wong, "Broad-Band Probe-Fed Patch Antenna With a U-shaped Ground Plane for Cross-Polarization Reduction," IEEE Trans. Antennas and Propag., vol. 50, no. 3, pp. 352-355, March 2002.

[9] X. Y. Zhang, J. X. Chen, Q. Xue and S. M. Li, "Dual-band Bandpass Filters Using Stub-Loaded Resonators," Micro. and Wireless Components Lett., vol. 17, No. 8, pp. 583-585, August 2007. 